\title{
VI. General method for determining the orbits of comets
}

\section{Laplace}

To cite this article: M. Laplace (1812) VI. General method for determining the orbits of comets , Philosophical Magazine Series 1, 39:165, 40-49, DOI: 10.1080/14786441208638080

To link to this article: http://dx.doi.org/10.1080/14786441208638080

曲 Published online: 27 Jul 2009.

Submit your article to this journal $\widetilde{ }$

Џ Article views: 2

Q View related articles $\sqsubset$ 
sons have been induced to inquire whether these names were imposed with propriety. But why should these changes be perpetually introduced into a science the business of which is with life; to which chemistry is subservient only in a remote degree? Diseases were cured before chemical remedies were invented. And where is this alteration of names to end? If the discoveries of Mr. Davy are established, the names of the alkalies are already absurd. Why might not these three substances be termed ammonia, kali, and barilla? They are very distinct, and convey to our minds no adventitious ideas. And the substances they designate may remain either in the vegetable or mineral kingdom, as future experience may determine, without any occasion to alter their denominations.

In looking into Duncan's late edition of the Pharmacopœia, we find five and even six names applied to one substance; some of which are current in London, others in Dublin, and another kind only is understood in Edinburgh. Suppose an individual were to pass by a different name in each of these capitals, the law would find it same difficulty in recognising him.

The suls and supers bear some resemblance to the aps and the macs and the o's, by which certain nations attempt to indicate family affinities, but every one knows the confounding of individual personality thence resulting; and that if you are in search of a Mr. O'Flannagan, you must not only designate him by his patronymic, but also his christian name, to which it is very requisite to add that of the county, the village, and the hamlet where he was born, and perbaps his numerical order in the series of births of his prolific progenitors.

ONOMOS.

VI. General Method for determining the Orbits of Comets. By M. LAPLACE *.

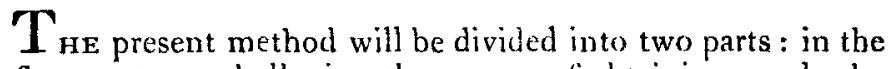
first part we shall give the means of oblaining nearly the perihelion distance, and the instant of the passage of the comet by this point : in the second part we sball determine precisely all the elements of the orbit, supposing the latter to be nearly known.

Accurate Determination of the Perihelion Distance, and of the Instant of the Passage of the Comet ly this Point.

1. We shall take three, four, five or more observations

* Translated from M. I aplace's Théorie du Mouvement et de la Figure des Planites, a work now become very scarce.-EDiT. 
of the comet equally as far as possible removed from each other: we should embrace within four observations an interval of 30 degrees, and within five observations an interval of 36 or 40 degrees, and so forth: but the interval comprehended between the observations must always be the larger the greater the number, in order to diminish the influence of their errors.

This being done, let $\beta, \beta^{\prime}, \beta^{\prime \prime}, \beta^{\prime \prime \prime}$, \&c. be the successive geocentric longitudes of the comet; $\gamma, \gamma^{\prime}, \gamma^{\prime \prime}, \gamma^{\prime \prime}$, \&c. the corresponding northern latitudes, the southern latitudes being supposed to be negative. Divide the difference $\beta^{\prime}-\beta$ by the number of days which separate the second from the first observation : divide in the same way the difference $\beta^{\prime \prime}-\beta^{\prime}$ by the number of days which separate the third from the second observation: divide also the difference $\beta^{\prime \prime \prime}-\beta^{\prime \prime}$ by the number of days which separate the fourth from the third observation, and so forth. Let $\delta \beta, \delta \beta^{\prime}, \delta \beta^{\prime \prime}$, $\delta \beta^{\prime \prime \prime}, \& \mathrm{c}$. be the consequence of these quotients.

Divide the difference $\delta \beta^{\prime}-\delta \beta$ by the number of days which separate the third from the first observation : divide in the same way the difference $\delta \beta^{\prime \prime}-\delta \beta^{\prime}$ by the number of days which separate the fourth from the second observation : divide also the difference $\delta \beta^{\prime \prime \prime}-\delta \beta^{\prime \prime}$ by the number of days which separate the fifth from the third observation, \&c. Let $\delta^{2} \beta, \delta^{2} \beta^{\prime}, \delta^{2} \beta^{\prime \prime}$ be the consequence of these quotients.

Divide the difference $\delta^{2} \beta^{\prime}-\delta^{2} \beta$ by the number of days which separate the fourth from the first observation : divide in the same way the difference $\delta^{2} \beta^{\prime \prime}-\delta^{2} \beta^{\prime}$ by the number of days which separate the fifth from the second obsertion, \&c. Let $\delta^{3} \beta, \delta^{3} \beta^{\prime}, \& c$. be the consequence of these quotients. We may go on in this way until we succeed in forming $\delta^{n-1} \cdot \beta, n$ being the number of the observations employed. This being accomplished,

2. Take a mean or nearly mean epoch between the instants of the two extreme observations, and by naming $i, i^{\prime}, i^{\prime \prime}, i^{\prime \prime \prime}, \& c$. the number of days by which it precedes every observation; $i, i^{\prime}$, \&c. being supposed to be negative for all the observations anterior to this epoch; the longitude of the comet after a small number $z$ of days counted from the epoch, will be expressed by the formula

$$
\begin{aligned}
& \beta-i . \delta \beta+i \cdot i^{\prime} \cdot \delta^{2} \cdot \beta-i . i^{\prime} \cdot i^{\prime \prime} \delta^{3} \cdot \beta+i \cdot i^{\prime} \cdot i^{\prime \prime} \cdot i^{\prime \prime \prime} \cdot \delta^{4} \cdot \beta-\& c \text {. } \\
& \begin{array}{c}
+z \cdot\left\{\begin{array}{c}
\delta \cdot \beta-\left(i+i^{\prime}\right) \cdot \delta^{2} \cdot \beta+\left(i \cdot i^{\prime}+i \cdot i^{\prime \prime}+i^{\prime} \cdot i^{\prime \prime}\right) \cdot \delta^{3} \cdot \beta \\
-\left(i i^{\prime} i^{\prime \prime}+i i^{\prime} i^{\prime \prime \prime}+i \cdot i^{\prime \prime} \cdot i^{\prime \prime \prime}+i^{\prime} \cdot i^{\prime \prime} \cdot i^{\prime \prime \prime}\right) \cdot \delta^{4} \cdot \beta \\
+\alpha c \cdot
\end{array}\right\} \ldots(p) \\
+z^{2} \cdot\left\{\begin{array}{c}
\delta^{2} \beta-\left(i+i^{\prime}+i^{\prime \prime}\right) \cdot \delta^{3} \cdot \beta+\left(i \cdot i^{\prime}+i \cdot i^{\prime \prime}+i \cdot i^{\prime \prime \prime}\right. \\
\left.+i^{\prime} \cdot i^{\prime \prime}+i^{\prime} \cdot i^{\prime \prime \prime}+i^{\prime \prime} \cdot i^{\prime \prime \prime}\right) \cdot \delta^{4} \beta-\alpha c .
\end{array}\right\}
\end{array}
\end{aligned}
$$

The coefficients of $-\delta . \beta,+\delta^{2} \beta$, \&c. in the part indepen- 
dent of $z$, are: 1 st, the number $i$; $2 \mathrm{~d}$, the product of the two numbers, $i$ and $i^{\prime} ; 3 \mathrm{~d}$, the products of the three num. bers $i, i^{\prime}, i^{\prime \prime}, \& c$.

The coefficients of $-\delta^{2} \cdot \beta,+\delta^{3} \cdot \beta,-\delta^{4} \cdot \beta, \& c$. in the part multiplied by $z$, are: 1 st, the sum of the two numbers $i$ and $i^{\prime} ; 2 \mathrm{~d}$, the sum of the products two by two, of the three numbers $i, i^{\prime}, i^{\prime \prime} ; 3 d$, the sum of the products three by three of the four numbers $i, i^{\prime}, i^{\prime \prime}, i^{\prime \prime \prime}, \& c$.

The coefficients of $-\delta^{3}, \beta,+\delta^{4} . \beta,-\delta^{5} . \beta, \& \mathrm{c}$. in the part multiplied by $z^{2}$, are: 1st, the sum of the three numbers $i, i^{\prime}, i^{\prime \prime} ; 2 \mathrm{~d}$, the sum of the products two by two of the four numbers $i, i^{\prime}, i^{\prime \prime}, i^{\prime \prime \prime} ; 3 \mathrm{~d}$, the sum of the products three by three of the five numbers $i, i^{\prime}, i^{\prime \prime}, i^{\prime \prime \prime}, i^{\prime \prime \prime \prime}, 8 \mathrm{cc}$.

By working in the same way on the latiludes of the comet, its latitude, according to the number $z$ of days since the epoch, will be expressed by the following formula,

$$
\begin{aligned}
& \gamma-i . \delta \gamma+i . i^{\prime} \cdot \delta^{8} \cdot \gamma-i \cdot i^{\prime} \cdot i^{\prime \prime} \cdot \delta^{3} \cdot \gamma+i . i^{\prime} \cdot i^{\prime \prime} \cdot i^{\prime \prime \prime} \cdot \delta^{4} \gamma-\& c . \\
& +z \cdot\left\{\begin{array}{c}
\delta \gamma-\left(i+i^{\prime}\right) \cdot \delta^{2} \gamma+\left(i \cdot i^{\prime}+i \cdot i^{\prime \prime}+i^{\prime} \cdot i^{\prime \prime}\right) \cdot \delta^{8} \gamma \\
-\left(i \cdot i^{\prime} \cdot i^{\prime \prime}+i \cdot i^{\prime} i^{\prime \prime \prime}+i \cdot i^{\prime \prime} \cdot i^{\prime \prime \prime}+i^{\prime} \cdot i^{\prime \prime} \cdot i^{\prime \prime \prime}\right) \cdot \delta^{4} \gamma \\
+8 c .
\end{array}\right\} \ldots(q) \\
& +z^{2} \cdot\left\{\begin{array}{r}
\delta^{2} \cdot \gamma-\left(i+i^{\prime}+i^{\prime \prime}\right) \cdot \delta^{s} \cdot \gamma+\left(i \cdot i^{\prime}+i \cdot i^{\prime}+i \cdot i^{\prime \prime \prime}\right. \\
\left.+i^{\prime} \cdot i^{\prime \prime}+i^{\prime} \cdot i^{\prime \prime \prime}+i^{\prime \prime} \cdot i^{\prime \prime \prime}\right) \cdot \delta^{4} \gamma-3 c .
\end{array}\right\}
\end{aligned}
$$

This being done, we shall have $a$ equal to the part independent of $z$ in the formula $(p)$.

By reducing into seconds the coefficieut of $z$, and subtracting from the logarithm of this number of seconds, the Jogarithm 3.5500072, we shall have the logarithm of a number which we shall designate by $a$.

By reducing into seconds the coefficient of $z^{2}$, by afterwards taking the logarithm of the double of this number of seconds, and subtracting from this logarithm the following, $1 \cdot 7855994$, we sball have the logarithin of a number which we shall designate by $b$.

We shall afterwards have $\theta$ equal to the part independent of $\boldsymbol{z}$ in the formula $(q)$.

By reducing into seconds the coefficient of $z$, in this formula, and by subtracting from it 3.5500072 , we shall have the logarithm of a number which we shall designate by $h$.

By reducing into seconds the coefficient of $z^{2}$ in this same formula, and by subtracting $1 \cdot 7855894$ from the logarithm of the double of this number of seconds, we shall have the logarithm of a number which we shall designate by $l$.

It is upon the precision of the values of $a, b, h$, and $l$, that the exactness of the following method depends; and as their formation is very simple, we must choose and multiply the observations so as to obtain them with all possible rigour. These quantities are the differentials $\left(\frac{d x}{d t}\right) ;\left(\frac{d^{2} \alpha}{d t^{2}}\right)$; 
$\left(\frac{d \theta}{d t}\right)$ and $\left(\frac{d^{2} \theta}{d t^{2}}\right)$, which we have expressed, for the sake of greater simplicity, by the foregoing letters.

If the number of observations is an odd one, we should fix the epoch at the instant of the mean observation, which will dispense with our calculating the parts independent of $z$, in the two preceding formulæ; for it is visible that these parts are respectively equal to the longitude and to the latitude of the mean observation.

In order to elucidate what has been said, by an example, we shall select the second comet discovered by M. Mechain in 1781 , and the orbit of which he calculated according to this method: the observations which this learned astronomer chose for this purpose, are referred to the same hour of the day, viz. $8^{\mathrm{h}} 29^{\prime} 44^{\prime \prime}$ mean time at Paris : the following are the observations :

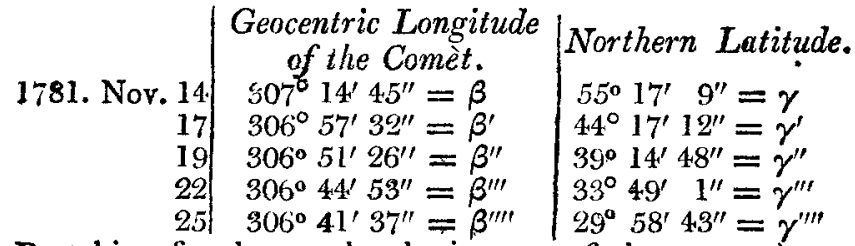

By taking for the epoch, the instant of the mean observation, $i$. e. the 19 th of November, at $8^{\text {h }} 29^{\prime} 44^{\prime \prime}$, we have which gives

$$
i=-5, i^{\prime}=-2, i^{\prime \prime}=0, i^{\prime \prime \prime}=3, i^{\prime \prime \prime \prime}=6 \text {, }
$$

$$
\begin{array}{c|l}
\delta \beta=-5^{\prime} 44^{\prime \prime}, 33 & \delta \gamma=-3^{0} 39^{\prime} 59^{\prime \prime}, 0 \\
\delta \beta^{\prime}=-3^{\prime} 3^{\prime \prime}, 0 & \delta \gamma^{\prime}=-2^{0} 31^{\prime} 12^{\prime \prime}, 0 \\
\delta \beta^{\prime \prime}=-2^{\prime} 11^{\prime \prime}, 0 & \delta \gamma^{\prime \prime}=-1^{\circ} 43^{\prime} 35^{\prime \prime}, 667 \\
\delta \beta^{\prime \prime \prime}=-1^{\prime} 5^{\prime \prime}, 53 & \delta \gamma^{\prime \prime \prime}=-1^{0} 16^{\prime} 46^{\prime \prime}, 0 \\
\hline \delta^{8} \beta=32^{\prime \prime}, 266 & \delta^{2} \gamma=13^{\prime} 45^{\prime \prime}, 4 \\
\delta^{2} \beta^{\prime}=10^{\prime \prime}, 4 & \delta^{2} \gamma^{\prime}=8^{\prime} 31^{\prime \prime}, 267 \\
\delta^{2} \beta^{\prime \prime}=10^{\prime \prime}, 945 & \delta^{8} \gamma^{\prime \prime}=5^{\prime} 18^{\prime \prime}, 278 \\
\hline \delta^{3} \beta=-2^{\prime \prime}, 733 & \delta^{3} \gamma=-39^{\prime \prime}, 26666 \\
\delta^{3} \beta^{\prime}=0^{\prime \prime}, 0631 & \delta^{3} \gamma^{\prime}=-24^{\prime \prime}, 1236 \\
\hline \delta^{4} \beta=0^{\prime \prime}, 2546 j & \delta^{4}=1^{\prime \prime}, 3766
\end{array}
$$

The formula $(p)$ will therefore give for the geocentric longitude of the comet, according to the small number $x$ of days reckoned from the epoch,

$$
306^{\circ} 51^{\prime} 26^{\prime \prime}-153^{\prime \prime}, 46 \cdot z+10^{\prime \prime}, 54 . z^{2} \text {, }
$$

and the formula $(q)$ will give for the expression of its latitude, from which we extract

$$
39^{\circ} 14^{\prime} 48^{\prime \prime}-7855^{\prime \prime}, 16 . z+535^{\prime \prime}, 4 . z^{2} \text {, }
$$

$$
\begin{gathered}
\alpha=306^{\circ} 51^{\prime} 26^{\prime \prime}, \\
\alpha=-0,0432501, l=0,345366, \\
\theta=39^{\circ} 14^{\prime} 48^{\prime \prime}, \\
h=-2,213844, l=17,54354 .
\end{gathered}
$$


2d. We shall determine by the astronomical tables the longitude of the earth seen from the sun at the instant which we have chosen for our epoch : let $A$ be the longitude, $R$ the corresponding distance from the earth to the sun, and $R^{\prime}$ the distance which answers to the longitude $90^{\circ}+A$, of the earth: we shall form the four equa. tions

$$
\begin{aligned}
& r^{2}=\frac{x^{2}}{\cos ^{2} \theta}+2 R x \cdot \cos (A-\alpha)+R^{2} \cdot \ldots(1) \\
y & =\frac{R \cdot \sin (A-\alpha)}{2 a} \cdot\left\{\frac{1}{R^{3}}-\frac{1}{r^{3}}\right\}-\frac{b x}{2 a} \ldots(2) \\
y & =-x \cdot\left\{h \cdot \operatorname{tang} \theta+\frac{l}{2 b}+\frac{a^{2} \cdot \sin \theta \cdot \cos \theta}{2 b}\right\} \\
& \left.+\frac{R \cdot \sin \theta}{2 h} \frac{\cos \theta}{2} \cdot \cos (A-x) \cdot\left\{\frac{1}{r^{9}}-\frac{1}{R^{3}}\right\}\right\} \ldots(3) \\
0 & =y^{2}+a^{2} \cdot x^{2}+\left\{y \cdot \operatorname{tang} \theta+\frac{b x}{\cos ^{2} \cdot \theta}\right\}^{2} \\
& \left.+2 y \cdot\left\{\left(R^{\prime}-1\right) \cdot \cos (A-\alpha)-\frac{\sin (A-\alpha)}{R}\right\}\right\} \ldots(4) \\
& +2 a x \cdot\left\{\left(R^{\prime}-1\right) \cdot \sin (A-\alpha)+\frac{\cos (A-\alpha)}{R}\right\} \\
& +\frac{1}{R^{2}}-\frac{2}{r} \cdot \ldots
\end{aligned}
$$

In order to draw from these equations the values of the three unknown quantities $x, y$, and $r$, we shall begin by considering if, abstraction being made of the sign, $b$ is greater or less than $l$; in the former case we shall make use of the equations (1), (2) and (4); we shall form a first hypothesis for $x$, by supposing it, for instance, equal to unity; and we shall extract from it by means of the equations (1) and (2), the values of $r$ and $y$; we shall afterwards substitute these values in the equation (4), and if the remains are null, it will be a proof that the value of $x$ has been well chosen; but if the remains are negative, we shall increase the value of $x$, and diminish it if the remains are positive. We shall also have by means of a small number of trials, the true values of $x, y$, and $r$; but as these unknown quantities may be susceptible of several values, we must choose that which satisfies precisely or nearly to the equation (3).

In the second case, $i$. $e$. if we have $l>b$, we shall make use of the equations (1), (3) and (4), and then it will be the equation (2) which will serve as the verification. 
Having thus the values of $x, y$, and $r$, we shall form the quantity

$$
\begin{aligned}
& P=\frac{x}{\cos ^{2} \theta} \cdot\{y+h x \cdot \operatorname{tang} \theta\}+R y \cdot \cos (A-\alpha) \\
& +x\left\{\left(R^{\prime}-1\right) \cdot \cos (A-\alpha)-\frac{\sin (A-\alpha)}{R}\right\} \\
& +\operatorname{Rax} \cdot \sin (A-\alpha)+R \cdot\left(R^{\prime}-1\right) .
\end{aligned}
$$

The perihelion distance $D$ of the comet will be

$$
D=r-\frac{1}{2} \cdot P^{2}
$$

the cosine of the anomaly $v$ of the comet will be given by the equation

$$
\cos ^{2} \frac{r}{2} v=\frac{D}{r}
$$

from which we shall conclude, by the table of the movement of the comets, the time consumed in traversing the angle $v^{*}$. In order to have the instant of the passage by the perihelion, we must add this time to the epoch, if $P$ is negative, and subtract it if $P$ is positive, because in the former case the comet approaches the perihelion, and in the second case it removes from it.

With respect to the second comet of 1781 , the epoch being fixed as above, on the 19th November, at $8^{\text {h }} 29^{\prime} 44^{\prime \prime}$, we have at this epoch

$$
\begin{gathered}
A=57^{\circ} 57^{\prime} 4^{\prime \prime}, \\
R=0,987248, R^{\prime}=0,988820,
\end{gathered}
$$

the equations (1), (2), (3) and (4) become thus

$$
\begin{gathered}
r^{2}=1,667387 \cdot x^{2}-0,7106137 . x+0,974653 \ldots(1) \\
y=-11,0665+\frac{10,6484}{r^{3}}+3,9927 . x \ldots(2) \\
y=5,771014 . x+\frac{0,03931687}{r^{3}}-0,04086053 \ldots(3) \\
0=y^{2}+0,00187057 \cdot x^{2} . \\
+[0,8169372 \cdot y-3,691334 \cdot x]^{2}-1,8320446 . y \ldots(4) . \\
\quad+0,0324357 . x+1,026006-\frac{2}{r} .
\end{gathered}
$$

* Call, as above, $D$ the perihelion distance of the comet, $U$ its anomaly, $i$. e.the angle formed by its vector radius with the axis of the parabola which it describes, lastly $t$, the time passed since the perihelion passage. This being done, according to the laws of parabolic motion, the time $t$ and the anomaly $U$ are united together by the following ties :

$$
\text { (1) } \ldots t=\frac{D^{\frac{3}{2}} T}{\pi \sqrt{2}}\left\{\text { tang } \frac{x}{2} U+\frac{5}{3} \operatorname{tang}^{3} \frac{t}{2} U\right\},
$$

in which $\pi$ is the demi-circumference, or 3,14159265 , and $T$ the duration of the sidereal revolution of the earth, or 365 days 256383 . $U$ being given, it is easy to calculate $t$ by this formula. But if $t$ is given, the search for the tang $\frac{2}{3} U$ requires the resolution of these equations of the $3 d$ degree. In or. der to avoid this difficulty, astronomers have formed a table of values of $t$ in a parabola in which $D$ will be equal to unity, and from this table when once calculated, we may extract the values of $U ; t$ being known. This is what we call a table of the motion of comets. We may supply the place of this table by resolving the equation (1) by some trials, 
As we have in this particular case $l_{7} b$, we must employ the equations (1), (3), and (4). These three equations give

$$
\begin{aligned}
& x=0,39107, \\
& y=2,258355, \\
& r=0,9755798 .
\end{aligned}
$$

These values satisfy the equation $(z)$ as much as we can expect of an equation which cannot be very exact on account of the little movement of the comet in longitude. By substituting them in the expression of $P$, we find

$$
P=-0,185628 \text {. }
$$

The negative sign of $P$ makes known that the comet has not yet attained its peribelion. We aftewards find the perihelion distance $D=0,9583509$, and the anomaly $v$ of the comet, equal to $15^{\circ} 16^{\prime} 24^{\prime \prime}$, which answers to 10 days 40334: from which it follows that the perihelion passage took place on the 29 th of November at $18^{\mathrm{h}} 10^{\prime} 34^{\prime \prime}$, mean time at Paris. Having thus obtained nearly the perihelion distance and the instant of the passage of the comet by this point, we may correct them by the following method, which has the advantage of being independent of an intimate knowledge of the other elements of the orbit.

Exact Determination of the Elements of the Orbit, when we know pretty nearly the Perihelion Distance and the Instant of the Passage of the Comet ly this Point.

3. We shall select three observations removed from the comet: by afterwards setting out from the perihelion distance, and from the instant of the passage by this point, determined by what precedes, we shall easily calculate the three anomalies of the comet, and the three vector radii corresponding to the instants of the three observations; let $v, v^{\prime}$ and $v^{\prime \prime}$ be these anomalies, those which precede the passage of the comet by the perihelion being necessarily supposed to be negative: further let $r, r^{\prime}, r^{\prime \prime}$ be the corresponding vector radii of the comet, $v^{\prime}-v$ and $v^{\prime \prime}-v$ will be the angles comprehended between $r$ and $r^{\prime}$ and between $r$ and $r^{\prime \prime}$; let $U$ be the first of these angles and $U^{\prime}$ the second.

Let us also call $a, a^{\prime}, a^{\prime \prime}$, the three geocentric longitudes observed of the comet; $\theta, \theta^{\prime}, \hat{\theta}^{\prime \prime}$, its three geocentric latitudes, the southern latitudes being supposed to be negative: $C, C^{\prime}, C^{\prime \prime}$, the three corresponding longitudes of the sun; $R, R^{\prime}, R^{\prime \prime}$, its three distances from the earth; $\beta, \beta^{\prime}, \beta^{\prime \prime}$, the three heliocentric longitudes of the comet; $\varpi, \varpi^{\prime \prime}, \varpi^{\prime \prime}$, its three heliocentric latitudes. This being done,

We will imagine the letter $S$ at the centre of the sun, the letter $T$ at the centre of the earth, the letter $C$ at the cenire 
of the comet, and the letter $C^{\prime}$ at its projection in the plane of the ecliptic; we shall have the angle $S T C^{\prime}$, on taking the difference of the geocentric longitudes of the sun and the comet; by afterwards adding the logarithm of the cosine of this angle with that of the cosine of the geocentric latitude $\theta$ of the comet, we shall have the logarithm of the cosine of the angle $S T C$; we shall therefure know in the triangle $S T C$, the side $S T$ or $R$, the side $S C$ or $r$, and the angle $S T C$; we shall thus have by rectilinear trigonometry the angle CST; we shall afterwards have the heliocentric latitude of the comet by means of the equation

$$
\sin w=\frac{\sin \theta \sin C S T}{\sin C T S} .
$$

The angle $T S C^{\prime}$ is the side of a spherical rectangular triangle, the hypothenuse of which is the angle TSC, and one of the sides of which is the angle ; from thence we shall easily extract the angle $T S C^{\prime}$, and consequently the heliocentric longitude $\beta$ of the comet.

We shall have in the same manner $\varpi^{\prime}, \beta^{\prime}, \varpi^{\prime \prime}$ and $\beta^{\prime \prime}$, and the values of $\beta, \beta^{\prime}, \beta^{\prime \prime}$ will show whether the motion of the comet be direct or retrograde.

If we conceive the two arcs of latitude $\approx$ and $w^{\circ}$ united at the pole of the ecliptic, they will there form an angle equal to $\beta^{\prime}-\beta$; and in the spherical triangle formed by this angle, and by the sides $90^{\circ}-\approx$ and $90^{\circ}-w^{\prime}$, the side opposite to the angle $\beta^{\prime}-\beta$ will be the angle at the sun comprehended between the two vector radii $r$ and $r^{\prime}$. We shall easily determine it by the known analogies of spherical trigonometry, or by the following formula :

$$
\cos V=\cos \left(\beta^{\prime}-\beta\right) \cdot \cos \pi \cdot \cos \pi^{\prime}+\sin \pi \cdot \sin \varpi^{\prime},
$$
in which $V$ represents this angle.

By calling $V^{\prime}$ in a similar manner the angle formed by the two vector radii $r$ and $r^{\prime \prime}$, we shall have

$$
\cos V^{\prime}=\cos \left(\beta^{\prime \prime}-\beta\right) \cdot \cos \pi \cdot \cos \pi^{\prime \prime}-\sin \pi \cdot \sin \varpi^{\prime \prime} .
$$

Now if the perihelion distance and the instant of the passage of the comet by this point were exactly determined, we shall have

$$
V=U \text { and } V^{\prime}=U^{\prime} \text {; }
$$

but as that will almost never happen, we shall suppose

$$
m=U-V ; n=U^{\prime}-V^{\prime} \text {. }
$$

We shall here observe that the calculation of the triangle $S T C$, gives for the angle $C S T$, two different values, viz. $C S T$ and $180^{\circ}$; $2 \mathrm{dly}, S T C-C S T$. We shail thus have two different values for each of the quantities $\beta$, $\varpi$, $\beta^{\prime}, \varpi^{\prime}, \beta^{\prime \prime}, w^{\prime \prime}$. Most frequently the nature of the motion 
of the comet will make known the value of CST, of which we ought to make use, particularly if these two angles are very different; for then one of them will place the comet further than the other from the earth, and it will be easy to ascertain by the apparent motion of the comet, at the instant of the observation, which of the two ought to be preferred. In a great number of cases, one of them will be negative, and must consequently be rejected; but if any uncertainty remains on this head, we might always determine the true values of $\beta, \beta^{\prime}, \beta^{\prime \prime}$, by observing to take for $\beta$ and $\beta^{\prime}$, the two angles which render $V$ very little different from $U$, and to take for $\beta$ and $\beta^{\prime \prime}$ the two angles which render $V^{\prime}$ very little different from $U^{\prime}$.

We shall afterwards form a second hypothesis, in which, by preserving the same instant of peribelion passage with the above, we shall vary the perihelion distance $t y$ a small quantity, for example, by the fiftieth part of its value, and we shall find out in this hypothesis the values of $U-V$, and of $U^{\prime}-V^{\prime}$; thus,

$$
m^{\prime}=U-V, n^{\prime}-U^{\prime}-V^{\prime}
$$

finally, we shall form a third hypothesis, in which, by preserving the same perihelion distance as in the first, we shall vary by half a day, or a whole day (more or less) the instant of the perihelion passage. We shall find out in this new hypothesis, the values of $U-V$ and of $U^{\prime}-V^{\prime}$; thus,

$$
m^{\prime \prime}=U-V, n^{\prime \prime}=U^{\prime}-V^{\prime} \text {; }
$$

this being done, if we call $u$ the number by which we ought to multiply the supposed variation in the perihelion distance in order to have the true one, and $t$ the number by which we ought to multiply the supposed variation in the instant of the perihelion passage in order to have the true instant, we shall have the two equations,

$$
\begin{aligned}
& u\left(m-m^{\prime}\right)+t\left(m-m^{\prime \prime}\right)=m, \\
& u\left(n-n^{\prime}\right)+t\left(n-n^{\prime \prime}\right)=n,
\end{aligned}
$$

from which we shall extract $u$ and $t$; and consequently the peribelion distance corrected and the true instant of the passage of the comet by this point.

The foregoing correction supposes that these elements determined by the first approximation, are sufficiently exact to treat as infinitely small their differences from the true: but if the second approximation did not still appear sufficient, we might have recourse to a third by operating on the elements already corrected, as has been done upon the first: we must only take care to make them undergo smaller variations. But in most cases this third approxi- 
mation will be useless, particularly if, in the first, we use four or five well selected observations.

We may also, in the correction of the first elements, make use of the second differences in the following manner.

Instead of calculating the values of $U, U^{\prime}, V$ and $V^{\prime}$ in the three hypotheses, we shall calculate them in five hypotheses, viz. 1. With the elements found by the first approximation. 2. By varying the perihelion distance by a very small quantity. 3. By varying it by double this small quantity. 4. By preserving the same peribelion distance as in the first hypothesis, and varying by a small interval the instant of the perihelion passage. 5. By varying the same instant double this interval. Let $n, m^{\prime}, m^{\prime \prime}$, $m^{\prime \prime \prime}, m^{\prime \prime \prime \prime}$ be the values of $U-V ; n, n^{\prime}, n^{\prime \prime}, n^{\prime \prime \prime}, n^{\prime \prime \prime \prime}$ the values of $U^{\prime}-V^{\prime}:$ then in order to determine the values of $u$ and $t$, we shall form the (wo equations,

$$
\begin{aligned}
\left(4 m^{\prime}-3 m-m^{\prime \prime}\right) \cdot u & +\left(m^{\prime \prime}-2 m^{\prime}+m\right) \cdot 2 t^{2}+\left(4 m^{\prime \prime \prime}-3 m-m^{\prime \prime \prime}\right) \cdot t \\
& +\left(m^{\prime \prime \prime}-2 m^{\prime \prime \prime}+m\right) \cdot t^{2}+2 m=0, \\
\left(4 n^{\prime}-3 n-n^{\prime \prime}\right) \cdot u & +\left(n^{\prime \prime}-2 n^{\prime}+n\right) \cdot u^{2}+\left(4 n^{\prime \prime \prime}-3 n-n^{\prime \prime \prime \prime}\right) \cdot t \\
& +\left(n^{\prime \prime \prime \prime}-2 n^{\prime \prime \prime}+n\right) \cdot t^{2}+2 n=0 .
\end{aligned}
$$

The values of $u$ and of $t$ which satisfy these equations will be more precise than the foregoing. Although in most cases this overmuch precision is useless, it is nevertheless indispensable in forming these equations, at all times when the terms depending on the second differences may be of the same order with those which depend on the first differences ; which will happen, for instance, when in one of the observations the vector radius of the comet will be almost perpendicular to the visual ray from the earth to the comet.

[To be continued.]

\section{A Case of Morbus Pedicularis. Communicated ly John Andree, Esq. Surgeon.}

A. R. esq. 35 years of age, of a very healthy and strong constitution, had for some years past, particularly when heated by good living and in warm rooms, been often troubled with a prickly itching on the surface of the body, armpits and thighs. In the summer of last year, on coming out of a warm bath at Brighton, he discovered an insect on his thigh.

This eircumstance induced him to suspect that the itching he had so long been troubled with, might have been caused by insects. From that time until he applied to me Vol. 39. No. 165. Jan. 1812. 